\title{
Towards oscillation-free implementation of the immersed boundary method with spectral-like methods
}

\author{
Jiannong Fang*, Marc Diebold, Chad Higgins, Marc B. Parlange \\ School of Architecture, Civil and Environmental Engineering (ENAC), École Polytechnique Fédérale de Lausanne (EPFL), CH-1015 Lausanne, Switzerland
}

\section{A R T I C L E I N F O}

\section{Article history:}

Received 14 January 2011

Received in revised form 31 May 2011

Accepted 8 July 2011

Available online $\mathrm{xxxx}$

\section{Keywords:}

Radial basis function

Immersed boundary method

Spectral method

Gibbs oscillation

Incompressible viscous flow

Navier-Stokes equations

Projection method

\begin{abstract}
A B S T R A C T
It is known that, when the immersed boundary method (IBM) is implemented within spectral-like methods, the Gibbs oscillation seriously deteriorates the calculation of derivatives near the body surface. In this paper, a radial basis function (RBF) based smoothing technique is proposed with the intention of eliminating or efficiently reducing the Gibbs oscillation without affecting the flow field outside the body. Based on this technique, a combined IBM/spectral scheme is developed to solve the incompressible Navier-Stokes equations. Numerical simulations of flow through a periodic lattice of cylinders of various cross sections are performed. The results demonstrate that the proposed methodology is able to give accurate and nearly oscillation-free numerical solutions of incompressible viscous flows.
\end{abstract}

(c) 2011 Elsevier Inc. All rights reserved.

\section{Introduction}

Immersed boundary methods (IBM) offer a simple strategy to use a regular computational grid while solving flow problems with complex geometry. Due to this advantage, IBM has gained popularity in many applications such as direct and large-eddy simulation [1-3]. The original idea of the immersed boundary method is to model the no-slip condition at the solid body surface by introducing a source term in the momentum equations, the so-called immersed boundary force, in such a way that the flow inside the solid domain is frozen $[4,5]$. IB methods can be classified into two main approaches. In the first approach [4-8], termed as the continuous forcing approach, the immersed boundary force is introduced into the partial differential equations for momentum before discretization. In the second approach [9-14], named the discrete forcing approach (also known as the direct forcing approach), the immersed boundary force is applied after the equations are discretized. Advantages and disadvantages of the two approaches were discussed in the review paper by Mittal and laccarino [15]. For flows without moving boundaries, the discrete forcing approach is more attractive because it enables a sharp representation of the immersed boundary and does not introduce additional numerical stability restriction.

Spectral-like methods such as the pseudo-spectral method and high-order compact schemes have been widely used in the field of computational fluid dynamics due to their very favorable accuracy properties. However, the spectral-like methods usually adopts a regular computational grid and can not be applied to problems with complex geometries. To remove this limitation, some attempts have been made to incorporate IBM to the spectral-like methods. When doing so, spurious oscillations occur in the calculated velocity derivatives near the immersed boundary. This is associated with the Gibbs phenomenon of spectral methods, i.e., the characteristic oscillatory behavior of the discrete Fourier series of a function with a

\footnotetext{
* Corresponding author. Tel.: +41 21693 2357; fax: +41216936330.

E-mail address: jiannong.fang@epfl.ch (J. Fang).
} 
discontinuity (even if the discontinuity concerns only the first derivative). Here, the discontinuity is introduced into the computational domain by the immersed boundary between the solid and the fluid. Because the Gibbs phenomenon is related to the slow decay of the Fourier coefficients of a discontinuous function, it is natural to reduce the oscillations by damping out the higher modes. Different spectral smoothing procedures are available [16], but all of them have side effect on the velocity field outside the solid body, i.e., loss of accuracy. An alternative approach is to represent a discontinuous function as the sum of a smooth function and a correction term determined by jump conditions [17]. This approach requires additional modifications of the spectral algorithm. Another approach is to reduce or modify the discontinuity near the immersed boundary. For the continuous forcing approach, Lamballais and Silvestrini [18] suggested spanning the immersed boundary force over 4-6 points with a gaussian weighting function centered at the wall. The price to pay is that the location of the wall becomes more diffuse. It was also found $[5,18]$ that applying the immersed boundary force only at the surface and leaving the fluid inside free can partly solve the problem. For the discrete forcing approach, Mohd-Yusof [9] suggests to specify a target velocity field in the solid domain by using a mirror flow of the fluid domain, i.e., estimating the inner velocities at the closest grid points to the body surface as the reserve to the outer velocities, thus creating a local stagnation point. By this way, the discontinuity is shifted into the solid body. However, for the spectral-like methods, such a modification would not be adequate for the purpose of reducing oscillation because of the non-local character of the derivative calculation. For the flow around a circular cylinder, Parnaudeau et al. [19] proposed an analytical formula for defining a smooth target velocity field in the full solid domain. A significant reduction of the oscillations was reported. Laplacian smoothing has also been tried [20,21], in which the velocity field inside the body is set by applying the Laplace operator iteratively. Nevertheless, the final smoothing effect strongly depends on the initial guess of the velocity and the number of iterations, for which a proper choice is often difficult to make.

The purpose of this paper is to develop a general smoothing technique for the implementation of IBM with the spectrallike methods aimed at eliminating or reducing efficiently the Gibbs oscillation without affecting the flow field outside the body. We will focus on the combination of the discrete forcing approach with the pseudo-spectral method. The outline of the paper is as follows. In the next section, we introduce a new smoothing procedure based on the radial basis function interpolation. In Section 3, we describe some combined IBM/spectral schemes including the novel scheme using the proposed smoothing technique. In Section 4, numerical tests are presented to validate the proposed approach. Finally, conclusions and remarks are given in Section 5.

\section{Radial basis function based smoothing}

A signed distance function $\varphi$ is used to identify the points inside the body $(\varphi \leqslant 0)$ and those in the flow domain $(\varphi>0)$. In general, we would like to approximate a variable, the velocity component $u(\boldsymbol{x})$ for example, inside the body in a smooth way without introducing a discontinuity. The idea proposed here is to interpolate the variable value at any point inside the body by using the known variable values at the points near the body surface and satisfying the boundary conditions. The interpolation formula is given as

$$
u(\boldsymbol{x})=\sum_{i=1}^{N} c_{i} \phi\left(\left\|\boldsymbol{x}-\boldsymbol{x}_{i}\right\|\right)
$$

where $c_{i}$ is the interpolation coefficient and $\phi$ is a radial basis function whose value depends only on the distance from the origin. Commonly used types of radial basis functions include Gaussian, Multiquadric, and Polyharmonic spline. The radial basis function adopted here is Gaussian, i.e.,

$$
\phi\left(\left\|\boldsymbol{x}-\boldsymbol{x}_{i}\right\|\right)=\exp \left(-\beta \frac{\left\|\boldsymbol{x}-\boldsymbol{x}_{i}\right\|^{2}}{d x^{2}}\right),
$$

where $d x$ is the grid space and $\beta$ is a positive parameter usually set to 0.5 . From the given variable values $u\left(\boldsymbol{x}_{i}\right)(i=1,2, \ldots, N)$, the unknown coefficients $c_{i}(i=1,2, \ldots, N)$ are obtained by solving the following linear algebra equations

$$
\boldsymbol{A c}=\boldsymbol{b},
$$

with

$$
\begin{aligned}
& \boldsymbol{b}=\left[u_{1}, u_{2}, \ldots, u_{N}\right]^{\mathrm{T}} \\
& \boldsymbol{c}=\left[c_{1}, c_{2}, \ldots, c_{N}\right]^{\mathrm{T}}
\end{aligned}
$$

and

$$
\boldsymbol{A}=\left(\begin{array}{cccc}
1 & \phi\left(\left\|\boldsymbol{x}_{1}-\boldsymbol{x}_{2}\right\|\right) & \ldots & \phi\left(\left\|\boldsymbol{x}_{1}-\boldsymbol{x}_{N}\right\|\right) \\
\phi\left(\left\|\boldsymbol{x}_{2}-\boldsymbol{x}_{1}\right\|\right) & 1 & \ldots & \phi\left(\left\|\boldsymbol{x}_{2}-\boldsymbol{x}_{N}\right\|\right) \\
\vdots & \vdots & \ddots & \vdots \\
\phi\left(\left\|\boldsymbol{x}_{N}-\boldsymbol{x}_{1}\right\|\right) & \phi\left(\left\|\boldsymbol{x}_{N}-\boldsymbol{x}_{2}\right\|\right) & \ldots & 1
\end{array}\right)
$$




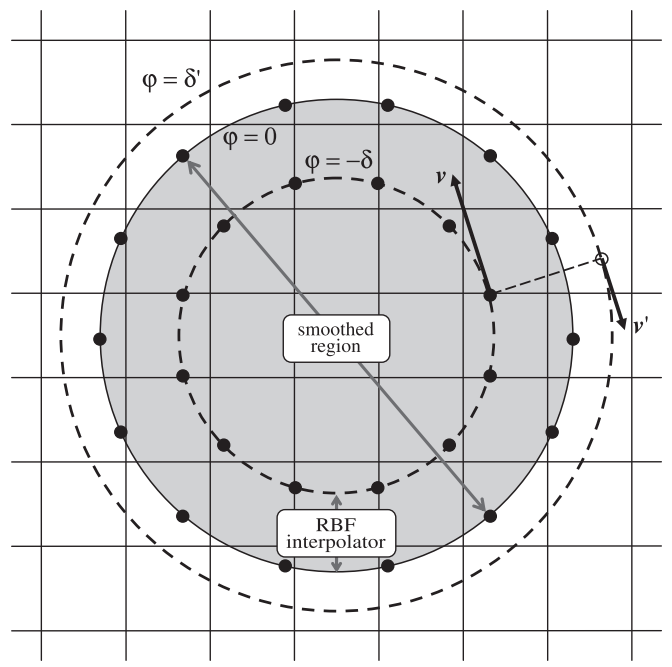

Fig. 1. Schematic showing the smoothing procedure of RBFS. Here, all the auxiliary points used for constructing the RBF interpolation are represented by solid circles.

To satisfy the no-slip condition at the body surface, two layers of auxiliary points are introduced. The first layer is on the body surface $(\varphi=0)$ where the velocity is known to be zero. The second layer is inside the body and at a distance $\delta$ to the surface $(\varphi=-\delta)$. The auxiliary points are arranged in such a way that the average spacing between them is also around $\delta$. First, the velocity at each auxiliary point of the second layer is obtained by a linear interpolation. Let $\boldsymbol{x}$ be the position of an auxiliary point and $\boldsymbol{n}$ denote the surface normal passing through $\boldsymbol{x}$. A corresponding point at a distance $\delta^{\prime}$ from the immersed boundary is defined as $\boldsymbol{x}^{\prime}=\boldsymbol{x}+\left(\delta+\delta^{\prime}\right) \boldsymbol{n}$. Let $\boldsymbol{v}$ be the velocity at the auxiliary point and $\boldsymbol{v}^{\prime}$ denote the velocity at the corresponding point. The linear interpolation gives $\boldsymbol{v}=-\boldsymbol{v}^{\prime} \delta / \delta^{\prime}$ in which zero velocity on the immersed boundary is applied. Then, the auxiliary points of the two layers are used to construct the RBF interpolator given by Eq. (1). Finally, the obtained RBF interpolator is applied repeatedly for all the regular grid points inside the body $(\varphi<0)$. Usually, the velocity at the corresponding point is not known and needs to be interpolated. In this paper, the trilinear finite element interpolation is used. Although the interpolation formula is simple and easy to implement, it could happen that some nodes of the interpolation element are located inside the body and hence their velocities are not known. Nevertheless, by using the velocity imaging relation for all internal nodes involved in the interpolation, a closed system of linear algebra equations can be obtained for the unknowns on these nodes and be solved by either direct or iterative method. A more efficient solution of the problem is to increase the distance $\delta^{\prime}$ to ensure that all the nodes of the interpolation elements locate outside the body. The RBF-based smoothing procedure described above, named as RBFS hereafter, is illustrated in Fig. 1.

To test the proposed smoothing technique, we here introduce a typical velocity profile around a solid body as shown in Fig. 2, where the velocity is usually set to zero inside the body. The velocity profiles obtained by applying different smoothing techniques are shown in Fig. 2, while the corresponding derivatives calculated by the pseudo-spectral method are presented in Fig. 3. It is observed that RBFS is more effective than the Laplacian smoothing in removing the Gibbs oscillations presented in the calculated derivatives.

\section{Combined IBM/spectral schemes}

In this section, a combined IBM/Spectral scheme using the smoothing technique proposed in the previous section will be developed for numerical simulation of unsteady incompressible viscous flow. The governing equations are the Navier-Stokes equations. When written in rotational form, they read

$$
\begin{aligned}
& \frac{\partial u_{i}}{\partial x_{i}}=0, \\
& \frac{\partial u_{i}}{\partial t}+u_{j}\left(\frac{\partial u_{i}}{\partial x_{j}}-\frac{\partial u_{j}}{\partial x_{i}}\right)=-\frac{\partial p}{\partial x_{i}}+v \frac{\partial^{2} u_{i}}{\partial x_{j} \partial x_{j}}+F_{i},
\end{aligned}
$$

where $t$ denotes the time, $u_{i}$ the $i$ th component of the fluid velocity, $x_{i}$ the $i$ th component of the position vector, $p$ is the effective kinematic pressure, $v$ the kinematic viscosity and $F_{i}$ the component of a body force such as gravity. The Einstein summation convention is used here, i.e. the summation is taken over repeated indices.

The projection method is adopted to solve Eqs. (4) and (5) in time. It consists of two fractional steps. At the first step the intermediate velocity is computed as follows: 


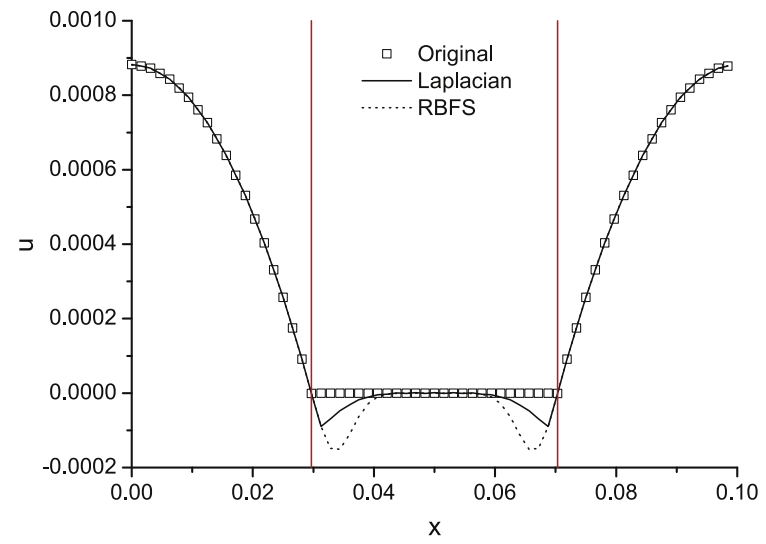

Fig. 2. The velocity profile constructed to resemble a flow as it passes across a bluff body represented by the two vertical lines and the profiles with the velocity inside the body smoothed by two different methods: Laplacian and RBFS.

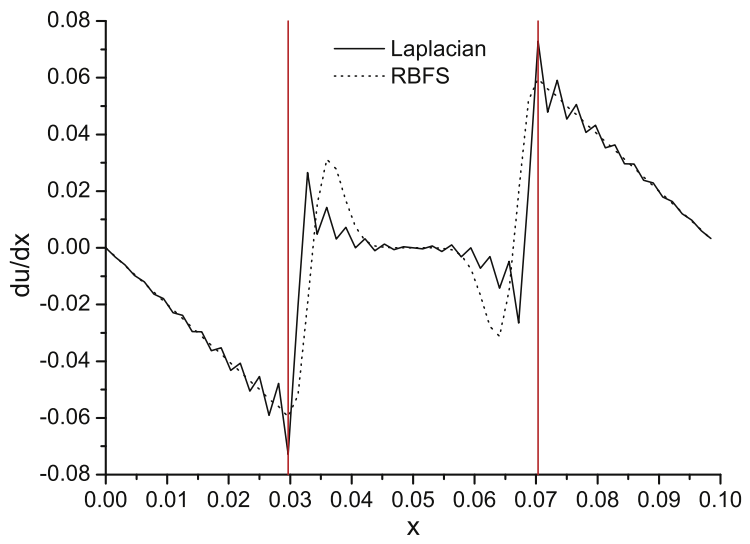

Fig. 3. The velocity derivative calculated by the pseudo-spectral method for the two smoothed velocity profiles in the previous figure.

$$
u_{i}^{*}=u_{i}^{n}+\frac{3}{2} \Delta t R_{i}^{n}-\frac{1}{2} \Delta t\left[R_{i}^{n-1}-\frac{\partial p^{n-1}}{\partial x_{i}}\right],
$$

where

$$
R_{i}^{n}=u_{j}^{n}\left(\frac{\partial u_{j}}{\partial x_{i}}-\frac{\partial u_{i}}{\partial x_{j}}\right)^{n}+v\left(\frac{\partial^{2} u_{i}}{\partial x_{j} \partial x_{j}}\right)^{n}+F_{i}^{n} .
$$

Eq. (6) is obtained by integrating the momentum equation forward in time using the second-order Adams-Bashforth scheme without the pressure gradient term at the $n$th level. Then, at the second step, $u_{i}^{*}$ is corrected to give the new velocity $u_{i}^{n+1}$ as

$$
u_{i}^{n+1}=u_{i}^{*}-\frac{3}{2} \Delta t \frac{\partial p^{n}}{\partial x_{i}}
$$

where the pressure gradient is added. Meanwhile, the new velocity must satisfy the incompressibility constraint. By taking the divergence of Eq. (7) and requiring that $u_{i}^{n+1}$ is a divergence free vector, we obtain the Poisson equation for the pressure

$$
\frac{\partial^{2} p^{n}}{\partial x_{i} \partial x_{i}}=\frac{2}{3 \Delta t} \frac{\partial u_{i}^{*}}{\partial x_{i}}
$$

Projecting the Eq. (7) on the outward unit normal vector $\boldsymbol{n}$ of the boundary $\Gamma$, we obtain the Neumann boundary condition for $p$, i.e,

$$
\left(\frac{\partial p}{\partial \boldsymbol{n}}\right)^{n}=-\frac{2}{3 \Delta t}\left[u_{i}^{n+1} n_{i}-u_{i}^{*} n_{i}\right]_{\Gamma} .
$$

Assuming $\boldsymbol{u} \cdot \boldsymbol{n}=0$ (non-penetration) on $\Gamma$, it gives 


$$
\left(\frac{\partial p}{\partial \boldsymbol{n}}\right)^{n}=\frac{2}{3 \Delta t}\left[u_{i}^{*} n_{i}\right]_{\Gamma}
$$

The spatial derivatives appearing in the above equations are computed by the pseudo-spectral method in the horizontal $x$ and $y$ directions, while in the vertical $z$ direction they are computed with the second-order centered finite difference scheme in a staggered grid formulation. The nonlinear convection terms are de-aliased in Fourier space by the 3/2 rule. The Poisson Eq. (8) for pressure with the Neumann boundary condition (10) on the top and bottom boundaries is solved by using the spectral transform in the horizontal and second-order finite differences in the vertical with a tridiagonal solver.

In the present study, the discrete-time momentum forcing, $f_{i}$, is applied to simulate the effects of obstacles in the flow. Accordingly, Eq. (7) is modified to

$$
u_{i}^{n+1}=u_{i}^{*}-\frac{3}{2} \Delta t \frac{\partial p^{n}}{\partial x_{i}}+\Delta t f_{i}^{n}
$$

where the force $\boldsymbol{f}$ only acts on points inside or on the surface of the obstacle and drives the fluid velocity to desired value on these points. Zero is a practical choice for the desired value. In this case, $\boldsymbol{f}$ can be obtained directly as

$$
f_{i}^{n}(\boldsymbol{x})= \begin{cases}(3 / 2)\left(\partial p^{n} / \partial x_{i}\right)-u_{i}^{*} / \Delta t & \text { if } \varphi(\boldsymbol{x}) \leqslant 0, \\ 0 & \text { if } \varphi(\boldsymbol{x})>0 .\end{cases}
$$

Since $\boldsymbol{f}$ is generally not divergence free, the Poisson equation for pressure should be changed to

$$
\frac{\partial^{2} p^{n}}{\partial x_{i} \partial x_{i}}=\frac{2}{3 \Delta t} \frac{\partial u_{i}^{*}}{\partial x_{i}}+\frac{2}{3} \frac{\partial f_{i}^{n}}{\partial x_{i}} .
$$

The above two equations are coupled and can be solved iteratively till the numerical solution for $p^{n}$ and $\boldsymbol{f}^{n}$ converges. This combined IBM/spectral scheme with simple treatment of the velocity inside the body is named as IBM-Simple hereafter. In addition to the inherent problem of Gibbs oscillation, another flaw of IBM-Simple is that it cannot satisfy the non-slip boundary condition exactly unless the grid points happen to be located on the body surface.

To improve IBM-Simple with respect to the two problems mentioned above, the desired velocity field inside the solid body is obtained by applying the new smoothing techniques proposed in Section 2. It is important to note that the desired velocity $\boldsymbol{u}^{n+1}$ obtained in this way is not divergence free anymore. Due to this fact, the incompressible constraint $\nabla \cdot \boldsymbol{u}^{n+1}=0$ must be discarded inside the body. To do this, a mass source/sink is introduced inside the body and the Poisson equation for pressure is modified to

$$
\frac{\partial^{2} p^{n}}{\partial x_{i} \partial x_{i}}=\frac{2}{3 \Delta t}\left(\frac{\partial u_{i}^{*}}{\partial x_{i}}-\varepsilon \frac{\partial u_{i}^{n+1}}{\partial x_{i}}\right)+\frac{2}{3} \frac{\partial f_{i}^{n}}{\partial x_{i}},
$$

where $\varepsilon=1$ inside the body and $\varepsilon=0$ otherwise. The artificial force is now obtained as

$$
f_{i}^{n}(\boldsymbol{x})= \begin{cases}(3 / 2)\left(\partial p^{n} / \partial x_{i}\right)-\left(u_{i}^{*}-u_{i}^{\text {desired }}\right) / \Delta t & \text { if } \varphi(\boldsymbol{x}) \leqslant 0, \\ 0 & \text { if } \varphi(\boldsymbol{x})>0 .\end{cases}
$$

The intermediate velocity inside the body can either remain unchanged as it is calculated from Eq. (6) or be smoothed by applying a similar smoothing technique. The former choice is adopted in this paper. The whole simulation procedure for the proposed new scheme of combined IBM/spectral method is summarized as follows:

(1) At the time level $n$ calculate the intermediate velocity $\boldsymbol{u}^{*}$ according to Eq. (6).

(2) Solve Eq. (14) for pressure to get $p^{n}$ with $\boldsymbol{f}^{n}$ and $\nabla \cdot \boldsymbol{u}^{n+1}$ from the previous iteration (the initial guesses for $\boldsymbol{f}^{n}$ and $\nabla \cdot \boldsymbol{u}^{n+1}$ could be zero and $\nabla \cdot \boldsymbol{u}^{*}$ respectively).

(3) Calculate the corrected velocity $\boldsymbol{u}^{n+1}$ outside the body from Eq. (7).

(4) Apply the smoothing technique (RBFS) to obtain the desired velocity $\boldsymbol{u}^{n+1}$ inside the body.

(5) Update the force $\boldsymbol{f}^{n}$ according to Eq. (15).

(6) Compute the mass source/sink $\nabla \cdot \boldsymbol{u}^{n+1}$ inside the body.

(7) Repeat steps 2-6 till the solution for $p^{n}, \boldsymbol{f}^{n}$ and $\boldsymbol{u}^{n+1}$ converges.

(8) Go to the next time level.

From now on, the new scheme is called as IBM-RBFS and the scheme of Mohd-Yusof [9] which has no velocity smoothing involved is denoted as IBM-NS. In IBM-NS, velocity reversing is performed for the inner grid points close to the body surface and the velocity field further inside the body is developed freely by solving the governing equations as outside. All the three schemes (IBM-Simple, IBM-NS and IBM-RBFS) are implemented in the EPFL-LES code, which was first developed at Johns Hopkins University [22-24] and then extended at EPFL [25-29].

Since the numerical scheme is essentially explicit, several time step constraints must be satisfied for numerical stability, including a Courant-Friedrichs-Lewy (CFL) condition, 


$$
\Delta t \leqslant 0.15 \frac{h}{U_{\max }}
$$

and a constraint due to viscous diffusion,

$$
\Delta t \leqslant 0.125 \frac{h^{2}}{v}
$$

where $U_{\max }$ is the maximum value of velocity for a given problem. If a body force is to be considered, we then have the additional constraint

$$
\Delta t \leqslant 0.25 \min _{\forall i} \sqrt{\frac{h}{F_{i}}}
$$

\section{Numerical tests}

In this section, we examine the validity of the combined IBM/spectral schemes presented in this paper for the numerical simulation of incompressible viscous flows. The 2D horizontal flow through a periodic lattice of cylinders in $x-y$ plane is taken as a benchmark problem for testing. It has been studied extensively as a simple model of flow through fibrous porous media. The computational domain of the problem consists of a single circular cylinder of radius $a$ and its associated volume within the lattice (see Fig. 4). Flow is driven by a body force $F$ along the $x$-direction. On the four sides of the square, periodic boundary conditions are applied to model an infinite periodic arrangement of cylinders. This makes the spectral method favorable for solving the problem. The 3D EPFL-LES code is used to simulate this 2D horizontal flow by imposing stress-free

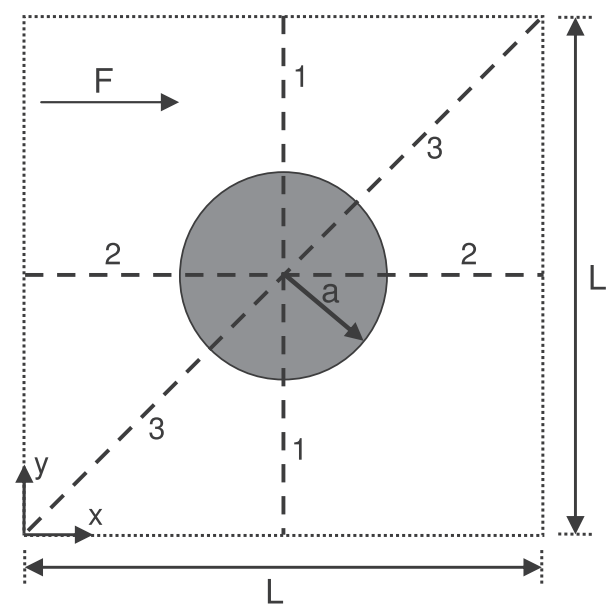

Fig. 4. Single cylinder within a periodic lattice and paths for comparison of numerical solutions.

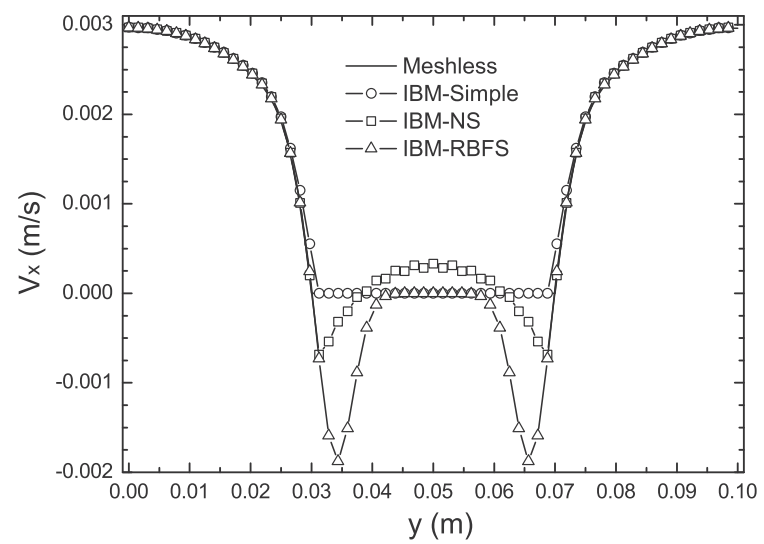

Fig. 5. Velocity profile along path 1 at $t=200 \mathrm{~s}$, predicted by IBM-Simple, IBM-NS, IBM-RBFS, and the meshless finite point method. 

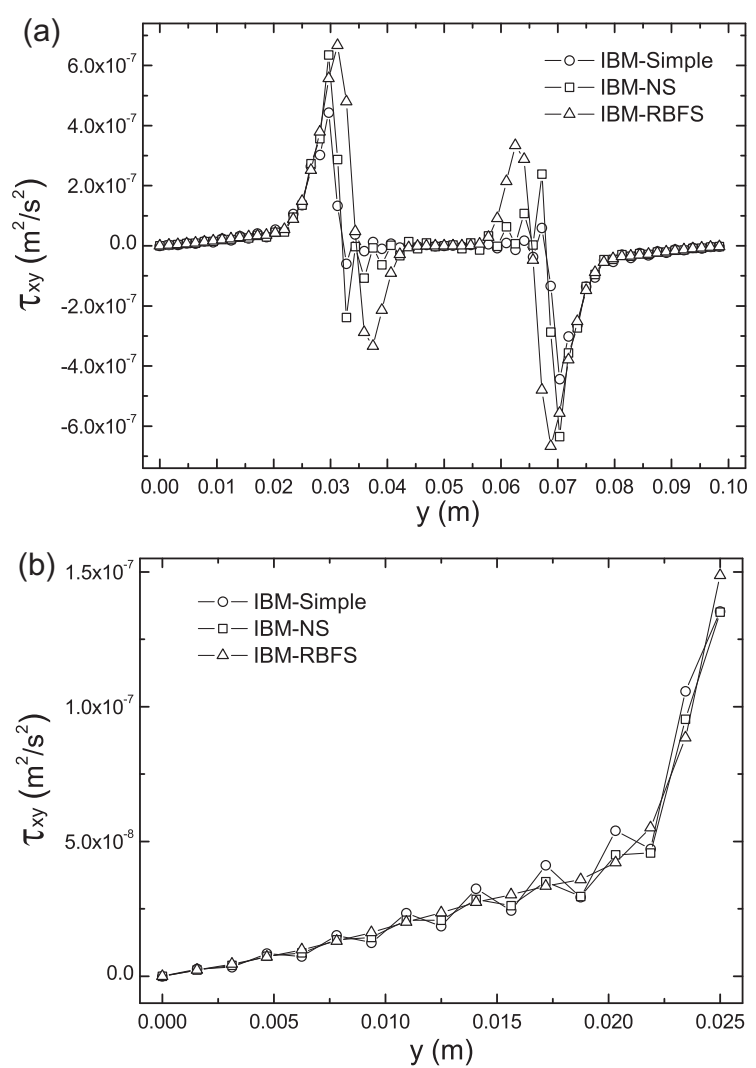

Fig. 6. (a) The shear stress component $\tau_{x y}$ along path 1 at $t=200 \mathrm{~s}$, predicted by IBM-Simple, IBM-NS and IBM-RBFS. (b) The left part of (a) enlarged.

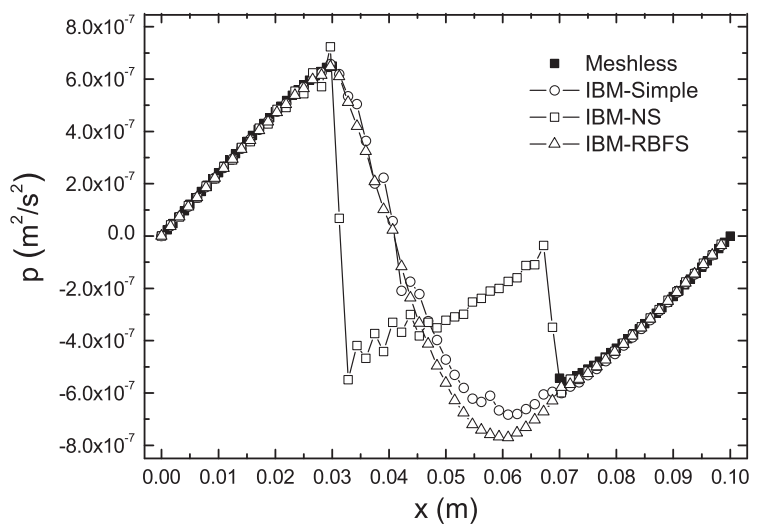

Fig. 7. Pressure profile along path 2 at $t=200 \mathrm{~s}$, predicted by IBM-Simple, IBM-NS, IBM-RBFS, and the meshless finite point method.

boundary conditions at the top and bottom of $z$. Hence, the discretizations along $x$ and $y$ in Fig. 4 are spectral and the discretization along $\mathrm{z}$ is finite difference but irrelevant here. On the boundary of the single cylinder a no-slip boundary condition is applied. The parameters of the geometry are $L=0.1 \mathrm{~m}$ and $a=0.02 \mathrm{~m}$. The kinematic viscosity is $v=10^{-6} \mathrm{~m}^{2} \mathrm{~s}^{-1}$ and the driven force is $F=1.5 \times 10^{-5} \mathrm{~ms}^{-2}$. The Reynolds number is defined as

$$
\operatorname{Re}=\frac{U a}{v}
$$

where $U$ is the characteristic velocity. For the case studied here, we have $U=5 \times 10^{-3} \mathrm{~ms}^{-1}$ which corresponds to Re $=100$.

Along path 1 defined in Fig. 4, the velocity profiles obtained by the three combined IBM/spectral schemes and a meshless finite point method [30] are compared in Fig. 5. The number of grid points is 64 for the plots here and similar plots after- 


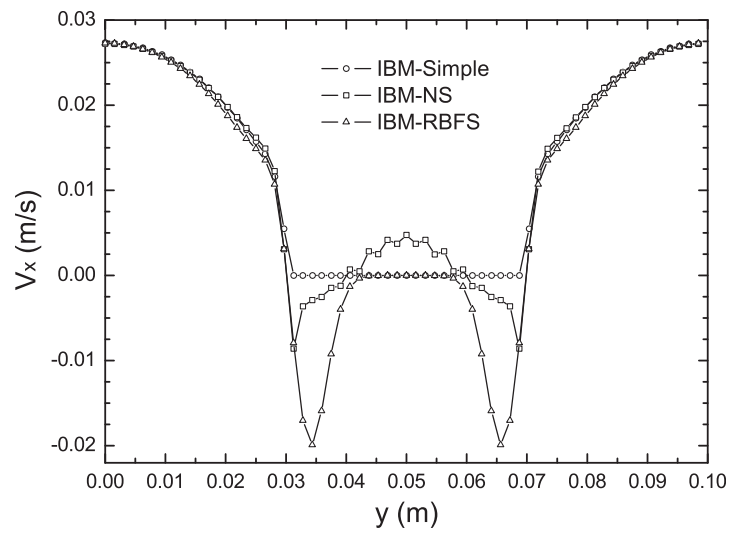

Fig. 8. Velocity profile along path 1 at $t=200 \mathrm{~s}$, predicted by IBM-Simple, IBM-NS and IBM-RBFS for Re $=1000$.
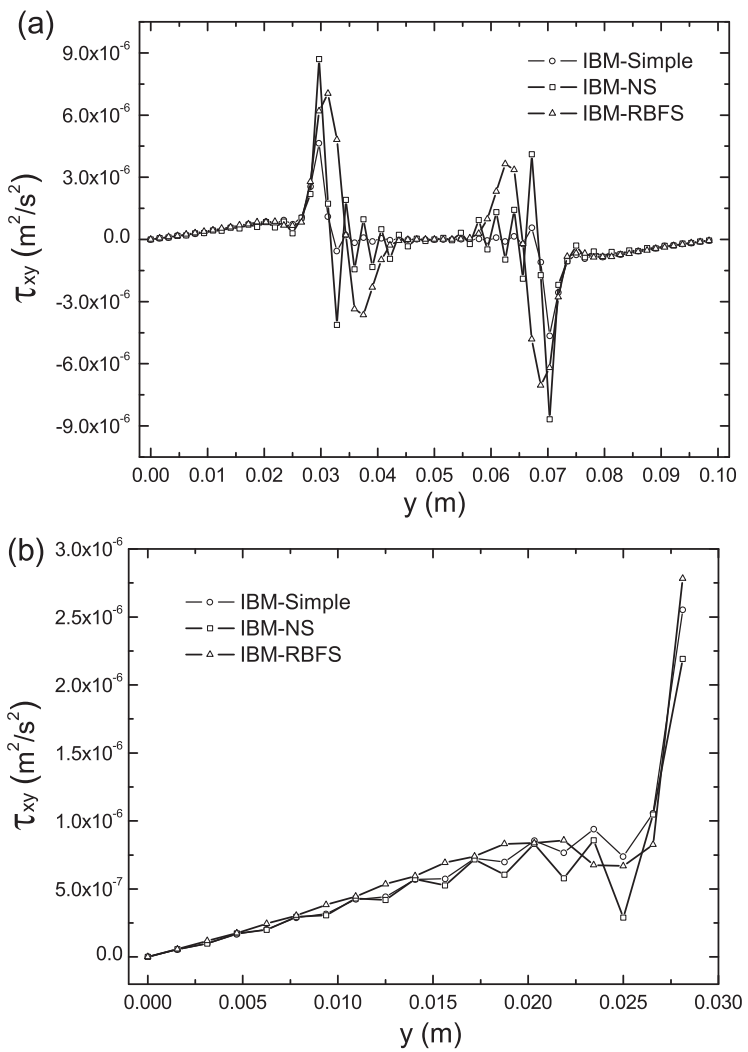

Fig. 9. (a) The shear stress component $\tau_{x y}$ along path 1 at $t=200 \mathrm{~s}$, predicted by IBM-Simple, IBM-NS and IBM-RBFS for Re $=1000$. (b) The left part of (a) enlarged.

wards, otherwise be specified explicitly. It can be seen that, outside the cylinder, the results of IBM-NS and IBM-RBFS are in good agreement with the meshless results, while IBM-Simple overpredicts the velocity near the cylinder surface because it does not satisfy exactly the non-slip boundary condition on the body surface. For both IBM-Simple and IBM-NS, Gibbs phenomenon of the spectral method has negligible influence on the predicted velocity outside the body. Nevertheless, small oscillation is observed inside the body for the velocity predicted by IBM-NS. Fig. 6 shows the results of the shear stress component $\tau_{x y}$ along path 1 . Here the shear stress component predicted by IBM-Simple and IBM-NS oscillates both inside and outside the body, while the result obtained by IBM-RBFS is almost oscillation free. In Fig. 7, the pressure fields obtained by different methods are compared along path 2 defined in Fig. 4 . It is found that the pressure result obtained by IBM-RBFS agrees well with that predicted by the meshless finite point method. The results by IBM-Simple and IBM-NS show significant oscillations, especially the pressure inside the cylinder predicted by IBM-NS. 


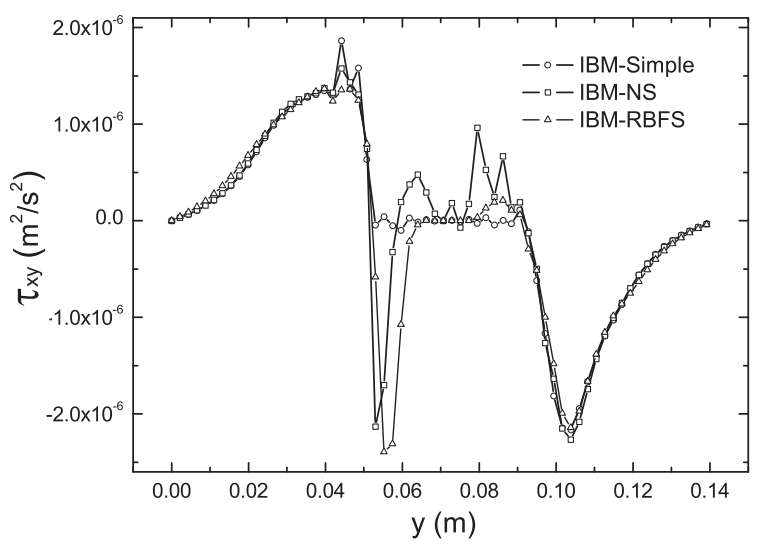

Fig. 10. The shear stress component $\tau_{x y}$ along path 3 ( 45 degrees with respect to the horizontal direction), predicted by IBM-Simple, IBM-NS and IBM-RBFS for $\operatorname{Re}=1000$.

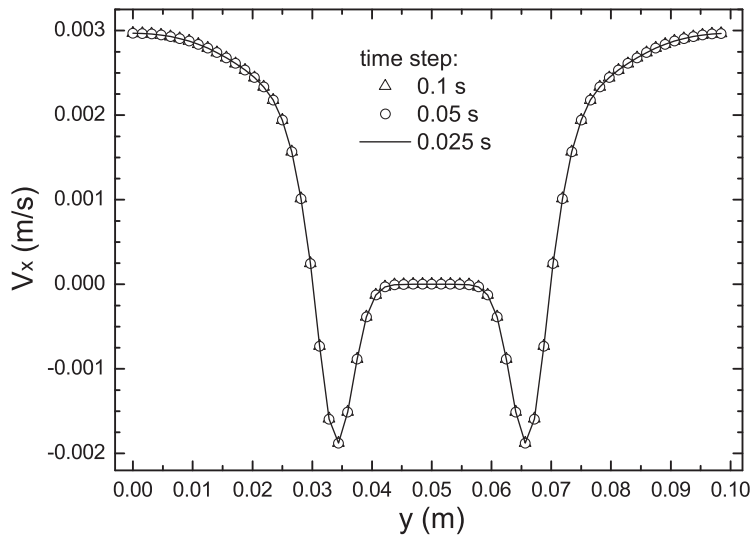

Fig. 11. Velocity profile along path 1 at $t=200 \mathrm{~s}$, predicted by IBM-RBFS with three different time steps.

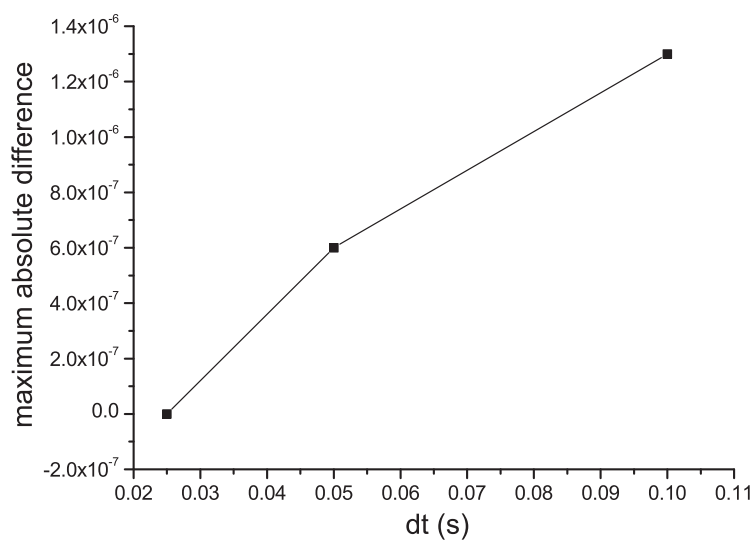

Fig. 12. The maximum absolute difference between the results of a given $\Delta t$ and those of $\Delta t=0.025 \mathrm{~s}$.

Simulation results obtained by different schemes at a higher Reynolds number $(\mathrm{Re}=1000)$ are compared in Figs. 8-10. Again, the three schemes (IBM-Simple, IBM-NS and IBM-RBFS) predict similar velocity profiles outside the cylinder, while IBM-RBFS yields much less oscillations in the shear stress profiles than the other two methods do.

Based on the results and observations presented above, it can be concluded that IBM-RBFS is superior to IBM-Simple and IBM-NS in terms of Gibbs oscillation alleviation. The increase of the total computational cost due to RBFS is about $20 \%$ over that of IBM-NS. The convergence of IBM-RBFS with respect to the grid space and the time step is studied here. Fig. 11 shows 


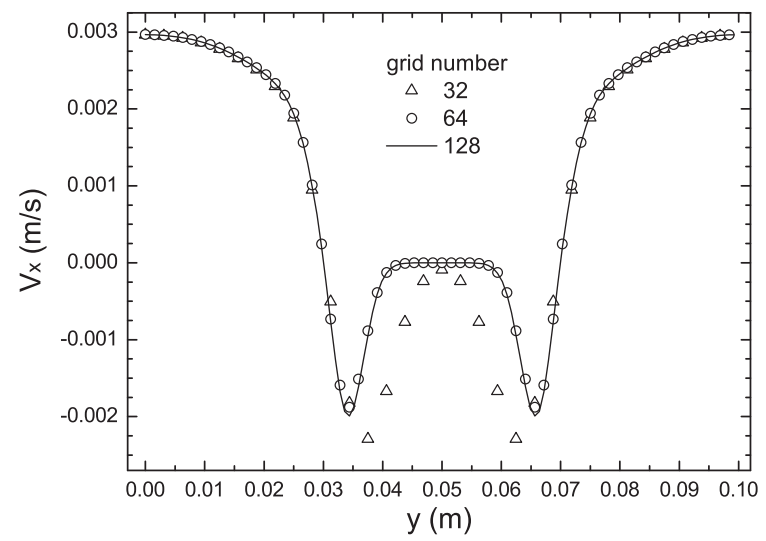

Fig. 13. Velocity profile along path 1 at $t=200 \mathrm{~s}$, predicted by IBM-RBFS with three different space resolutions.

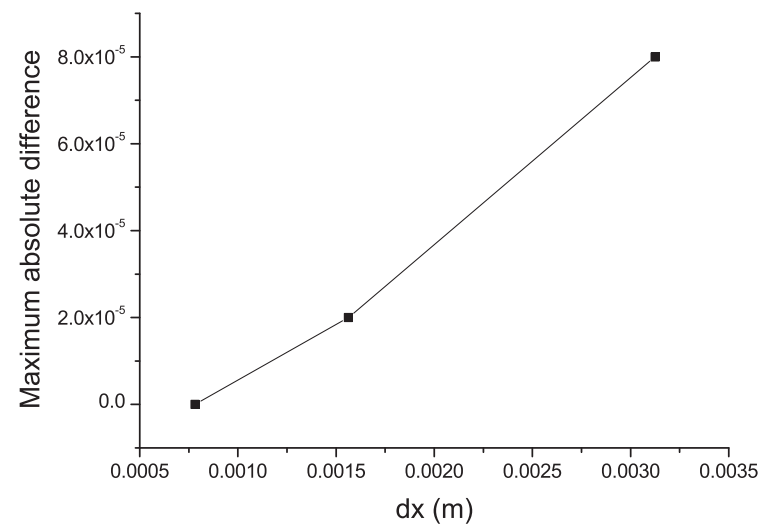

Fig. 14. The maximum absolute difference between the averaged horizontal velocity predicted with a given grid space and that predicted with the finest grid space.

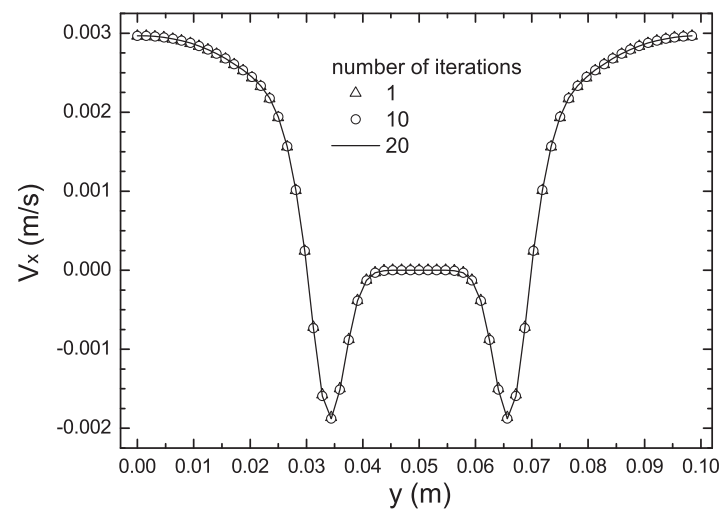

Fig. 15. Velocity profile along path 1 at $t=200 \mathrm{~s}$, predicted by IBM-RBFS with the coupled pressure and force equations solved iteratively using different number of iterations.

the velocity profiles along path 1 obtained with three different values of $\Delta t$. It can be seen that the results of different time steps almost overlap with each other. To analyze the convergence quantitatively, the maximum absolute difference between the results of a given $\Delta t$ and those of the smallest $\Delta t$ is plotted in Fig. 12. The decreasing curve indicates a trend of first-order convergence of IBM-RBFS with respect to the time step. The velocity profiles of three different space resolutions are shown in Fig. 13. Again, the three curves coincide with each other outside the cylinder. In Fig. 14, the maximum absolute difference between the averaged horizontal velocity predicted with a given grid space and that predicted with the finest grid space is 


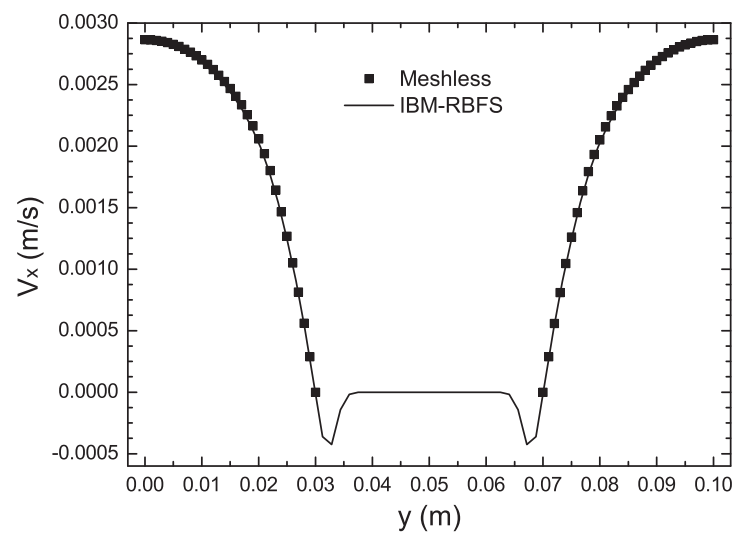

Fig. 16. Velocity profile along path 1 at $t=200 \mathrm{~s}$ for flow past the square cylinder, predicted by IBM-RBFS and the meshless finite point method.

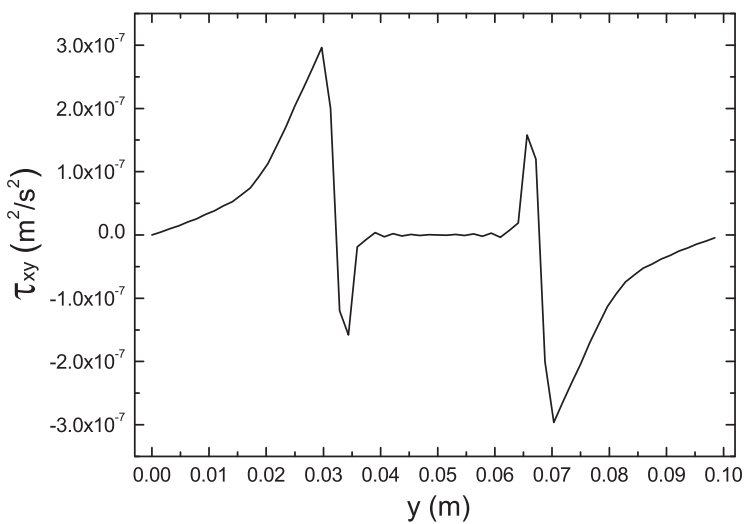

Fig. 17. The shear stress component $\tau_{x y}$ along path 1 at $t=200 \mathrm{~s}$ for flow past the square cylinder, predicted by IBM-RBFS.

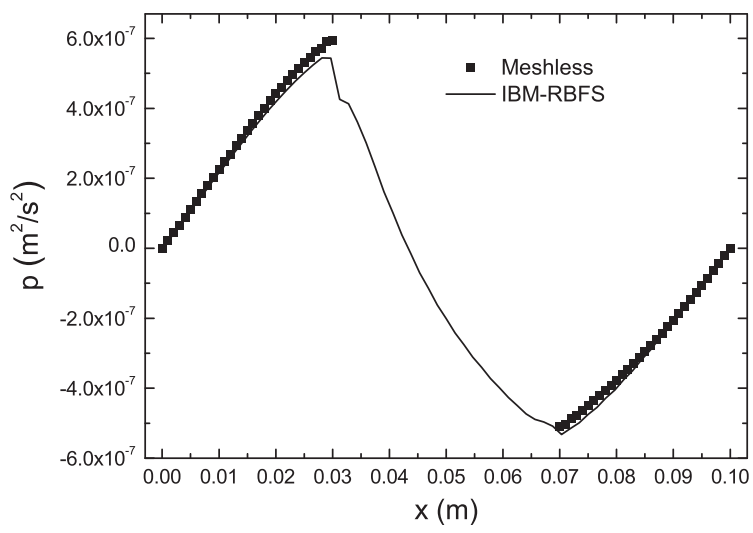

Fig. 18. Pressure profile along path 2 at $t=200 \mathrm{~s}$ for flow past the square cylinder, predicted by IBM-RBFS and the meshless finite point method.

shown. The decreasing difference with increasing space resolution confirms the convergence of IBM-RBFS with respect to the grid space.

When the immersed boundary technique is coupled with a projection method for the solution of the incompressible flow, which is the case in this paper and many others, incompressibility is enforced through the pressure field and no-slip condition on the solid wall is enforced through the immersed boundary force. These two processes interact with each other, To satisfy both requirements exactly, the iteration method proposed in Section 3 of this paper was applied. Compared to the extended project method [13], it is simpler for computer implementation, however, the computational cost would be higher 
if a large number of iterations needs to be performed for comparable accuracy. To explore on this issues, the velocity profiles obtained with different number of iterations for solving the coupled pressure and force equations are plotted in Fig. 15. It is shown that the differences between the results of few iterations and those of many iterations are quite small. Therefore, few iterations (even only one iteration) can be adopted for the sake of computational efficiency.

To show that the proposed smoothing technique works for bodies of more general surface geometry, the circular cylinder in the previous problem is replaced by a square cylinder of side length $0.04 \mathrm{~m}$ and the flow is simulated by IBM-RBFS with the other parameters remaining the same. To compare numerical results, path 1 and path 2 are defined similarly as in Fig. 4 . The velocity profile and the shear stress profile along path 1 are shown in Figs. 16 and 17 respectively. Again, it is found that the velocity profile predicted by IBM-RBFS is in good agreement with that obtained by the meshless finite point method and the shear stress profile obtained by IBM-RBFS is almost oscillation-free. Fig. 18 shows the pressure profile along path 2, from which, we can see that the results of IBM-RBFS match the meshless results quite well.

\section{Conclusions}

A radial basis function based smoothing technique (RBFS) is proposed in this paper with the intention of overcoming the oscillation problem associated with the Gibbs phenomenon of spectral approximation, which is encountered typically in the implementation of the immersed boundary method with spectral-like methods. Unlike analytical smoothing formula, RBFS can be applied for bodies of general surface geometry. Compared to the spectral smoothing approach, RBFS has no side-effect on the velocity field outside the body. It is easier to implement RBFS than to implement a global description of discontinuous functions. Based on it, a combined IBM/Spectral method (IBM-RBFS) is developed for the numerical simulation of incompressible flows. The projection method is adopted to satisfy the incompressibility constraint and the discrete-time immersed boundary forcing is applied to enforce the no-slip constraint on body surface. An iteration procedure is proposed to satisfy the two constraints exactly at each time step. Numerical simulations of the flow through a periodic lattice of cylinders are presented to validate the proposed methodology. The results indicate that IBM-RBFS is an accurate and robust numerical method for solving the incompressible Navier-Stokes equations. The convergence of IBM-RBFS with respect to time step and grid space is confirmed numerically. Compared to the other IBM implementations without the same kind of smoothing, the Gibbs oscillation is significantly alleviated by the proposed method. It is also found that, to reduce the computational cost, a small number of iterations can be adopted to solve the coupled pressure and forcing equations without deteriorating the final results. Although the proposed smoothing technique is only applied to the velocity field in this paper, it can be easily extended for other physical variables such as temperature for heat transfer, concentration for scalar transport, and subgridscale stress components for large eddy simulation, by making minor modifications to meet different boundary conditions on the body surface.

\section{Acknowledgements}

This research work has been partially supported by the Swiss National Science Foundation (Grant numbers: 200020125092, 200021-107910). Calculations were carried out on the Pleiades cluster at EPFL and the Cray XT5 system at the Swiss National Supercomputing Centre.

\section{References}

[1] R. Verzicco, J. Mohd-Yusof, P. Orlandi, D. Haworth, Large eddy simulation in complex geometric configurations using boundary body forces, AIAA J. 38 (2000) 427-433.

[2] A. Cristallo, R. Verzicco, Combined immersed boundary/large-eddy-simulations of incompressible three dimensional complex flows, Flow Turbulence Combust. 77 (2006) 3-26.

[3] E. Bou-Zeid, J. Overney, B.D. Rogers, M.B. Parlange, The effects of building representation and clustering in large-eddy simulations of flows in urban canopies, Bound. Layer Meteorol. 132 (2009) 415-436.

[4] C.S. Peskin, Flow patterns around heart valves: a numerical method, J. Comput. Phys. 10 (1972) $220-252$

[5] D. Goldstein, R. Handler, L. Sirovich, Modelling a no-slip boundary condition with an external force field, J. Comput. Phys. 105 (1993) 354-366.

[6] M.-C. Lai, C.S. Peskin, An immersed boundary method with formal second-order accuracy and reduced numerical viscosity, J. Comput. Phys. 160 (2000) 705-719.

[7] B.E. Griffith, C.S. Peskin, On the order of accuracy of the immersed boundary method: higher order convergence rates for sufficiently smooth problems, J. Comput. Phys. 208 (2005) 75-105.

[8] R.P. Beyer, R.J. LeVeque, Analysis of a one-dimensional model for the immersed boundary method, SIAM J. Numer. Anal. 29 (1992) $332-364$.

[9] J. Mohd-Yusof, Combined immersed-boundary/B-spline methods for simulations of flow in complex geometries, in: CTR Annual Research Briefs, Center for Turbulence Research, NASA Ames/Stanford Univ., 1997, pp. 317-327.

[10] E.A. Fadlun, R. Verzicco, P. Orlandi, J. Mohd-Yusof, Combined immersed-boundary finite-difference methods for three-dimensional complex flow simulations, J. Comput. Phys. 161 (2000) 35-60.

[11] J. Kim, D. Kim, H. Choi, An immersed-boundary finite-volume method for simulations of flow in complex geometries, J. Comput. Phys. 171 (2001) 132150.

[12] Y.-H. Tseng, J.H. Ferziger, A ghost-cell immersed boundary method for flow in complex geometry, J. Comput. Phys. 192 (2003) 593-623.

[13] K. Taira, T. Colonius, The immersed boundary method: a projection approach, J. Comput. Phys. 225 (2007) $2118-2137$.

[14] R. Mittal, H. Dong, M. Bozkurttas, F.M. Najjar, A. Vargas, A. von Loebbecke, A versatile sharp interface immersed boundary method for incompressible flows with complex boundaries, J. Comput. Phys. 227 (2008) 4825-4852.

[15] R. Mittal, G. Iaccarino, Immersed boundary methods, Ann. Rev. Fluid Mech. 37 (2005) 239-261.

[16] C. Canuto, M.Y. Hussaini, A. Quarteroni, T.A. Zang, Spectral Methods in Fluid Dynamics, Springer, Berlin, 1988. 
[17] A. Liang, X. Jing, X. Sun, Constructing spectral schemes of the immersed interface method via a global description of discontinuous functions, J. Comput. Phys. 227 (2008) 8341-8366.

[18] E. Lamballais, J. Silvestrini, Direct numerical simulation of interactions between a mixing layer and a wake around a cylinder, J. Turbul. 3 (2002) 028.

[19] P. Parnaudeau, E. Lamballais, D. Heitz, J. Silvestrini, Combination of the immersed boundary method with compact schemes for DNS of flows in complex geometry, in: R. Friedrich, B. Geurts, O. Mtais (Eds.), Direct and Large-Eddy Simulation V, ERCOFTAC Series, vol. 9, Kluwer Academic Publishers., Netherlands, 2004, pp. 581-590.

[20] Y.-H. Tseng, C. Meneveau, M.B. Parlange, Modeling flow around bluff bodies and predicting urban dispersion using large eddy simulation, Environ. Sci. Technol. 40 (8) (2006) 2653-2662.

[21] S. Chester, C. Meneveau, M.B. Parlange, Modeling turbulent flow over fractal trees with renormalized numerical simulation, J. Comput. Phys. 225 (1) (2007) 427-448.

[22] J.D. Albertson, M.B. Parlange, Surface length scales and shear stress: implications for land-atmosphere interaction over complex terrain, Water Resour. Res. 35 (7) (1999) 2121-2132.

[23] J.D. Albertson, M.B. Parlange, Natural integration of scalar fluxes from complex terrain, Adv. Water Resourc. 23 (3) (1999) $239-252$.

[24] F. Porté-Agel, C. Meneveau, M.B. Parlange, A scale-dependent dynamic model for large-eddy simulation: application to a neutral atmospheric boundary layer, J. Fluid Mech. 415 (2000) 261-284.

[25] E. Bou-Zeid, C. Meneveau, M.B. Parlange, A scale-dependent Lagrangian dynamic model for large eddy simulation of complex turbulent flows, Phys. Fluids 17 (2) (2005) 025105.

[26] V. Kumar, J. Kleissl, C. Meneveau, M.B. Parlange, Large-eddy simulation of a diurnal cycle of the atmospheric boundary layer: atmospheric stability and scaling issues, Water Resourc. Res. 42 (2006) W06D09.

[27] N. Vercauteren, E. Bou-Zeid, M.B. Parlange, H. Lemmin, H. Huwald, J. Selker, C. Meneveau, Subgrid-scale dynamics of water vapour, heat and momentum over a lake, Bound. Layer Meteorol. 128 (2) (2008) 205-228.

[28] D. Nadeau, V. Kumar, C. Higgins, M.B. Parlange, E. Pardyjak, Large-eddy simulation of the convective atmospheric boundary layer over heterogeneous land surfaces, in: American Geophysical Union Annual Meeting, San Francisco, USA, 2009.

[29] M. Calaf, C. Meneveau, J. Meyers, Large eddy simulation study of fully developed wind-turbine array boundary layers, Phys. Fluids 22 (1) (2010) 020001PHF.

[30] J. Fang, A. Parriaux, A regularized Lagrangian finite point method for the simulation of incompressible viscous flows, J. Comput. Phys. 227 (2008) $8894-$ 8908. 